\title{
Malva Sylvestris, Vitis Vinífera e Punica Granatum: uma revisão sobre a contribuição para o tratamento de periodontite
}

\author{
Malva Sylvestris, Vitis Vinífera e Punica Granatum: a review of the contribution to the treatment periodontitis
}

Malva Sylvestris, Vitis Vinífera y Punica Granatum: una revisión sobre la contribución para el tratamiento de la periodontitis

\author{
José Henrique de Araújo Cruz $^{1}$ \\ José Lucas Soares FERREIRA ${ }^{1}$ \\ André Paulo Gomes SIMÕES ${ }^{1}$ \\ Daniela Lima CRISTINO ${ }^{1}$ \\ Edivan Ilton Dantas da COSTA ${ }^{1}$ \\ Elaine Roberta Leite de SOUZA ${ }^{1}$ \\ Iolanda Alves de Oliveira DANTAS ${ }^{1}$ \\ Laise da Luz RAMOS ${ }^{1}$ \\ Nílvia Maria Lima GOMES ${ }^{1}$ \\ Raquel Lira Braga da SILVA \\ Abrahão Alves de OLIVEIRA FILHO²
}

\begin{abstract}
${ }^{1}$ Graduando do Curso de Odontologia, Universidade Federal de Campina Grande (UFCG), Campus Patos 58708-110 Patos - PB, Brasil ${ }^{2}$ Professor Doutor, Departamento de Odontologia, Curso de Odontologia, Universidade Federal de Campina Grande (UFCG), Campus Patos 58708-110 Patos - PB, Brasil
\end{abstract}

\section{Resumo}

As plantas medicinais têm demonstrado elevado poder de cura em estado natural, além disso, esse conhecimento tradicional sobre o uso das plantas e de suas propriedades terapêuticas no combate a doenças vêm sendo transmitida entre as gerações. A busca por novos produtos com maior atividade terapêutica, tem estimulado a realização de pesquisas com produtos naturais no meio odontológico para o tratamento de doença periodontal. Logo, objetivou-se apresentar uma revisão da literatura de espécies vegetais como Malva Sylvestris, Vitis Vinífera e Punica Granatum, comuns do cotidiano no tratamento da periodontite. A periodontite é uma doença inflamatória crônica decorrente da resposta imunológica do hospedeiro à presença de fatores microbianos, causando dano tecidual, resultando em formação de bolsas periodontais, reabsorção do osso alveolar, e perda de tecidos de sustentação. O estudo trata-se de uma revisão bibliográfica do tipo narrativa e foi realizada uma seleção de artigos científicos recuperados a partir das bases de dados: BVS Brasil (Biblioteca Virtual em Saúde), Scielo (Scientific Eletronic Library Online), Pubmed (National Center for Biotechnology Information) e Portal Periódico Capes no período de 05 a 28 de Fevereiro de 2018. Conclui-se que a Malva, Uva e Romã possuem ação terapêutica e estão entre os fitoterápicos com grande influência na cavidade bucal, que funcionam como auxiliares no tratamento de afecções orais sendo alternativas de fácil acesso, já que a atuação profissional frente à ação farmacológica dos vários medicamentos fitoterápicos e contraindicações tem sido importante nos últimos anos.

Descritores: Fitoterapia; Plantas Medicinais; Periodontite.

\begin{abstract}
Medicinal plants have shown high healing power in the natural state, and this traditional knowledge about the use of plants and their therapeutic properties in the fight against diseases has been transmitted between the generations. The search for new products with greater therapeutic activity has stimulated research with natural products in the dental environment for the treatment of periodontal disease. Therefore, it was aimed to present a review of the literature of plant species such as Malva Sylvestris, Vitis Vinífera and Punica Granatum, common daily in the treatment of periodontitis. Periodontitis is a chronic inflammatory disease resulting from the host's immune response to the presence of microbial factors, causing tissue damage, resulting in formation of periodontal pockets, alveolar bone resorption, and loss of supporting tissues. The study is a bibliographical review of the narrative type and a selection of scientific articles retrieved from the databases: VHL Brasil (Virtual Health Library), Scielo (Scientific Electronic Library Online), Pubmed (National Center for Biotechnology Information) and Portal Periódico Capes from 05 to 28 February 2018. It is concluded that Malva, Grape and Pomegranate have therapeutic action and are among herbal medicines with great influence in the oral cavity, which function as auxiliaries in the treatment of oral diseases being alternatives of easy access, since the professional action against the pharmacological action of the various herbal medicines and contraindications has been important in recent years.
\end{abstract}

Descriptors: Phytotherapy; Medicinal, Plants; Periodontitis.

\section{Resumen}

Las plantas medicinales han demostrado un elevado poder de curación en estado natural, además, ese conocimiento tradicional sobre el uso de las plantas y de sus propiedades terapéuticas en el combate a enfermedades vienen siendo transmitidas entre las generaciones. La búsqueda de nuevos productos con mayor actividad terapéutica, ha estimulado la realización de investigaciones con productos naturales en el medio odontológico para el tratamiento de enfermedad periodontal. Por lo tanto, se objetivó presentar una revisión de la literatura de especies vegetales como Malva Sylvestris, Vitis Vinífera y Punica Granatum, comunes de lo cotidiano en el tratamiento de la periodontitis. La periodontitis es una enfermedad inflamatoria crónica derivada de la respuesta inmunológica del huésped a la presencia de factores microbianos, causando daño tisular, resultando en formación de bolsas periodontal, reabsorción del hueso alveolar, y pérdida de tejidos de sustentación. El estudio se trata de una revisión bibliográfica del tipo narrativa y se realizó una selección de artículos científicos recuperados a partir de las bases de datos: BVS Brasil (Biblioteca Virtual en Salud), Scielo (Scientific Eletronic Library Online), Pubmed (National Center for Y se encuentra entre los fitoterápicos con gran influencia en la cavidad bucal, que funcionan como auxiliares en el tratamiento de la enfermedad de Chagas, en el período de 5 a 28 de febrero de 2018. Se concluye que Malva, Uva y Román poseen acción terapéutica y están entre los fitoterápicos con gran influencia en la cavidad bucal, que funcionan como auxiliares en el tratamiento de afecciones orales siendo alternativas de fácil acceso, ya que la actuación profesional frente a la acción farmacológica de los diversos medicamentos fitoterápicos y contraindicaciones ha sido importante en los últimos años.

Descriptores: Fitoterapia; Plantas Medicinales; Periodontitis.

\section{INTRODUÇÃO}

Os conhecimentos sobre a utilização de plantas no combate a doenças vêm sendo transmitida de maneira empírica entre as gerações e, as plantas medicinais têm demonstrado elevado poder de cura em estado natural, além disso, esse conhecimento tradicional sobre o uso das plantas e de suas propriedades terapêuticas, em alguns casos, é o único recurso disponível que a população rural de países 
em desenvolvimento possui ${ }^{1,2,3}$. No nordeste brasileiro, por exemplo, é comum a utilização de preparos caseiros de origem vegetal para o tratamento de várias enfermidades ${ }^{4}$.

Segundo a Organização Mundial da Saúde (OMS) $85 \%$ da população de países em desenvolvimento faz uso de plantas medicinais como forma de cuidado médico 5 . Segundo a literatura, estima-se que cerca de $40 \%$ dos medicamentos atuais são de origem natural ${ }^{6}$. As plantas medicinais produzem substâncias nomeadas metabólitos secundários que têm finalidade de fornecer melhor adaptação ao meio e promover a propagação da espécie vegetal. Esses metabólitos podem facilitar a polinização ou fornecer proteção contra predadores liberando odores, conferindo cor à planta ou atuando em nível celular protegendo contra invasores ${ }^{7}$.

Como exemplo de metabólitos secundários, temos os alcaloides, os terpenos e os flavonoides ${ }^{7}$. Estes metabólitos são descritos na literatura apresentando várias atividades biológicas, como atividade anti-inflamatória, antioxidante, antiparasitária $^{8,9,10}$.

A busca por novos produtos com maior atividade terapêutica, menor toxicidade e melhor biocompatibilidade tem estimulado a realização de pesquisas com produtos naturais no meio odontológico para o tratamento de doenças comuns da cavidade oral, como é o caso da doença periodontal $^{11}$.

Apesar de promissora a prática da medicina alternativa na odontologia, a fitoterapia é ainda pouco explorada, além de muitas vezes não ser inserida nos projetos pedagógicos dos cursos de graduação. Em virtude da busca por novas alternativas terapêuticas para periodontite e buscando capacitar profissionais de odontologia diante de suas contribuições, o presente trabalho objetivou apresentar uma revisão da literatura de espécies vegetais como Malva Sylvestris, Vitis Vinífera e Punica Granatum, comuns do cotidiano e suas propriedades terapêuticas no tratamento da periodontite.

\section{MATERIAL E MÉTODO}

O presente estudo trata-se de uma revisão bibliográfica do tipo narrativa, no qual foi realizada uma seleção de artigos científicos recuperados a partir das bases de dados: BVS Brasil (Biblioteca Virtual em Saúde), Scielo (Scientific Eletronic Library Online), Pubmed (National Center for Biotechnology Information) e Portal Periódico Capes no período de 05 a 28 de Fevereiro de 2018.

A estratégia de busca utilizada para pesquisa nas bases de dados se deu pela utilização dos seguintes descritores para Fisiopatologia da Periodontite: "Fisiopatologia da periodontite", "mediadores inaflamatórios", "metaloproteinases", "óxido nítrico". Para Malva Sylvestres: "periodontite", "odontologia", "fitoterapia". Para Vitis Vinífera: "Vitis Vinífera", "periodontite", "periodontitis", "dentistry", "odontologia". Para Punica Granatum: "Punica Granatum", "periodontite", "periodontitis", "romã". Seguindo a pesquisa de acordo com os descritores, nas bases de dados: Portal Periódico Capes, Scielo, BVS e PubMed, e utilizando como critérios de inclusão trabalhos publicados em português e inglês no período de 2000 a 2018, o número de artigos foi delimitado para 6325. Destes 6325, 52 artigos foram utilizados para produção desta revisão.

\section{RESULTADOS E DISCUSSÃO}

\section{- Fisiopatologia da Periodontite}

A periodontite é uma doença inflamatória crônica decorrente da resposta imunológica do hospedeiro à presença de fatores microbianos, na qual bactérias ativam as células de defesa formando um infiltrado leucocitário, e este na tentativa de combater o agente invasor, liberam mediadores inflamatórios que serão os responsáveis pelo dano tecidual, resultando em formação de bolsas periodontais, reabsorção do osso alveolar, e perda de tecidos de sustentação ${ }^{12}$.

A resposta imuno-inflamatória ocorre na tentativa de evitar a invasão e a disseminação de microrganismos nos tecidos orais, tendo em vista que essa reação de defesa do hospedeiro reflete prejuízo aos próprios tecidos, e atingindo de forma mais profunda o tecido conjuntivo, ultrapassando o sulco gengival, tem-se como resultado a perda de inserção das fibras colágenas e consequentemente, o osso alveolar sofre reabsorção, caracterizando a periodontite $^{13}$.

Os principais mediadores inflamatórios envolvidos na fisiopatologia da periodontite são as citocinas (IL-1, IL-6, IL-8, TNF-) as prostaglandinas (formada pela Cox1 e Cox 2) as metaloproteinases (MMP) e o óxido nítrico (NO). Todas elas refletem seus efeitos na cavidade oral. As citocinas, IL-1 e TNF-, por exemplo, estimulam a reabsorção óssea, já as prostaglandinas contribuem principalmente na vasodilatação e no aumento da permeabilidade $\operatorname{vascular}^{12}$. As metaloproteinases demonstram que durante as doenças inflamatórias agem na destruição das fibras colágenas ${ }^{14}$. O NO já se faz presente na cavidade oral em pequenas concentrações, funcionando como mecanismo de defesa natural contra os patógenos, mas em altas concentrações contribuem para destruir os tecidos orais ${ }^{15}$.

O papel principal dos PMN é a proteção, entretanto estes podem lançar uma gama de fatores que vão danificar os tecidos, incluindo espécies reativas de oxigênio, colagenases e outras proteases ${ }^{16}$.

Tendo em vista os efeitos dos mediadores inflamatórios e a influência deles na gravidade e extensão da doença periodontal, demonstra-se que a 
periodontite é resultado da defesa do hospedeiro aos fatores locais, e que a busca por substâncias que modulem a resposta inflamatória ou por agentes antimicrobianos que atuem no biofilme, tem funcionado como ferramenta de controle da periodontite ${ }^{17}$.

\section{- Malva Sylvestris}

A Malva Sylvestris é uma espécie de planta medicinal nativa de países europeus, pertencente à família Malvacea, com hábito herbáceo, podendo apresentar-se como planta ereta ou decumbente. A literatura cita-a como mucilaginosa, com propriedade adstringente e capacidade de reduzir inflamações ${ }^{18}$. Possui diversos componentes químicos como as vitaminas A, B1 e B2, óleos essenciais, ácido cumarínico, rica em carotenos e têm como principais constituintes os flavonoides e taninos ${ }^{19}$. A literatura também relata que os flavonoides e taninos são os princípios ativos responsáveis pela ação antiinflamatória da planta ${ }^{20}$.

A Malva Sylvestris tem diversos benefícios à saúde, e vem sendo bastante utilizada em tratamentos quimioterápicos, anti-inflamatórios, anti-cancros, em diversos tipos de infecções principalmente em áreas de mucosas como a mucosa vaginal e bucal ${ }^{21}$. O seu uso como planta medicinal vem ganhando espaço e se tornando cada dia mais utilizada no tratamento de diversos problemas de saúde, inclusive da saúde bucal, pois suas propriedades são importantes recursos para o tratamento de diversas patologias da cavidade bucal ${ }^{22}$.

No mercado nacional encontram-se substâncias antissépticas a base de Tintura de Malva, Quinosol e Tirotricina, que atuam nos processos inflamatórios orais. Sua denominação comercial é Malvatricin (Daudt), sendo indicada para aftas, gengivites, amidalites, faringites, estomatites e abscessos dentários. Utiliza-se $10 \mathrm{ml}$ da solução dissolvida em meio copo de água morna, para bochechos ou gargarejos três a quatro vezes ao $\operatorname{dia}^{23}$. Tem eficácia comprovada para bactérias que compõe o biofilme periodontopatogênico sendo assim considerado um medicamento fitoterápico eficaz, com propriedades anti-inflamatórias e antibióticas, além de possuir baixo custo, baixos níveis de toxicidade e boa biocompatibilidade ${ }^{21}$.

Com isso, sugere-se que mais estudos clínicos sobre a Malva Sylvestris precisem ser realizados para confirmar a hipótese de que ela possua eficácia como planta fitoterápica no tratamento de problemas como periodontite, por apresentar-se como um forte agente antimicrobiano, com atividade citotóxica que auxilia no controle do biofilme, agente etiológico primário da periodontite.

\section{- Vitis Vinífera}

A videira é uma planta trepadeira com tronco retorcido. Os frutos são bagas reunidas em cachos, onde se localizam as sementes, variando de cor de acordo com o tipo de uva ${ }^{24,25}$. Conforme a sua classificação botânica, pertence ao reino Plantae, filo Magnoliophyta, classe Magnoliopsida, ordem Vitales, família Vitaceae, gênero Vitis e contêm inúmeras espécies, sendo as mais conhecidas Vitis Vinífera e Vitis Labrusca, originárias, respectivamente, da Ásia e dos Estados Unidos ${ }^{26,27}$.

A literatura tem demonstrado que os resíduos de uva são ricos em compostos bioativos, especialmente polifenóis conhecidos por suas propriedades antioxidantes, inibindo ou retardando reações oxidativas e produzindo efeitos benéficos ao organismo humano, principalmente no que se refere à prevenção e/ou controle de doenças crônicas ${ }^{28-34}$. Os polifenóis apresentam-se em maiores concentrações na casca, semente e polpa da uva ${ }^{35}$.

Os vinhos tintos diferenciam-se dos brancos pela presença dos compostos fenólicos, que apresentam ação benéfica à saúde principalmente em razão das propriedades antioxidantes, bactericidas e vitamínicas, auxiliando na prevenção de doenças ${ }^{36}$. Dentre estes compostos estão o resveratrol, a quercetina, as catequinas e as proantocianidinas, que apresentam propriedades cardioprotetoras, anticancerígenas, anti-inflamatórias e antimicrobianas, devido principalmente à sua atividade antioxidante e anti-radicalar ${ }^{37}$.

As propriedades antioxidantes adjacentes de compostos químicos protegem as células do stress oxidativo, promovendo desta forma a saúde humana ${ }^{38}$. As diferentes classes de compostos fenólicos presentes no vinho ou na videira demonstram um elevado potencial antioxidante, sendo esta a mais notável bioatividade, que consiste na proteção do organismo mediante o sequestro de radicais livres e na inibição da oxidação lipídica ${ }^{38-40}$.

Rayyan et. al. ${ }^{41}$ avaliaram a eficácia da aplicação de um gel à base do extrato da semente de Vitis Vinífera em bolsas periodontais para o tratamento de periodontite crônica de pacientes sistemicamente saudáveis. Os resultados demonstraram melhoras significativas no índice de placa e índice gengival. De acordo com Barreto ${ }^{42}$ a realização de bochechos do suco concentrado ou mascar a uva (Vitis sp.) auxilia no tratamento de processos inflamatórios bucais.

Portanto, sugere-se que o bagaço da uva pode ter influência sobre a doença periodontal, especificamente a periodontite, uma vez que uma de suas causas são as atividades oriundas do estresse oxidativo mediante a resposta inflamatória, promovendo diminuição dos radicais livres produzidos diariamente durante as funções vitais das células. No entanto, mais estudos clínicos precisam ser realizados para confirmar esta hipótese.

- Punica Granatum

A romãzeira (Punica Granatum) é um arbusto grande, com ramificações espinhosas, da 
família Punicaceae, oriundo de uma região que compreende desde o Irã até o Himalaia, a noroeste da Índia. Há muito tempo vem sendo cultivada por toda a região Mediterrânea da Ásia, América, África e Europa. Suas folhas são de pequeno diâmetro, apresentando certa rigidez, brilhantes e membranáceas. As flores são de coloração vermelhoalaranjadas dispostas nas extremidades dos ramos que eventualmente originará os frutos, onde estes possuem formato arredondado, com muitas sementes de coloração esbranquiçada ao vermelho bordô, organizadas em camadas as quais se acham envolvidas em arilo membranoso ${ }^{43,44}$.

Tal planta, rica em compostos fenólicos, tem apresentado um grande potencial farmacológico como agente anti-inflamatório, antioxidante, antimicrobiano, antifúngico e antiviral demonstrado tanto em estudos in vitro quanto in vivo ${ }^{45-47}$.

A romã possui em sua composição compostos fenólicos como alcaloides, flavonoides, antocianinas, ácidos fenólicos e taninos, sendo este último responsável pela sua ação antibacteriana. Diversos autores também demonstraram em seus estudos que os flavonoides são os principais compostos presentes na romã responsáveis pela sua atividade anti-inflamatória ${ }^{48,49}$.

Sastravaha et al. ${ }^{50}$ avaliaram extratos herbais no tratamento periodontal, incluindo o pericarpo da Punica Granatum. Sob a forma de chips biodegradáveis, os extratos foram colocados em bolsas periodontais e associados à raspagem e aplainamento radicular em pacientes adultos. No grupo que foi tratado com os extratos, obteve-se melhora na reparação tecidual.

Bhadhaden et al. ${ }^{51}$ realizaram estudo in vitro onde buscou avaliar o efeito de um enxaguatório bucal contendo romã, determinar se ocorreria algum efeito adverso e suas propriedades terapeuticas sobre bacterias periodontopatogênicas. Como resultado, observou-se que o enxaguatório a base de romã tinha eficácia antibacteriana contra Aggregatibacter actinomycetemcomitans, Porphyromonas gingivalis e Prevotella intermedia que são os agentes patogênicos periodontais mais importantes associados à periodontite. Este enxaguatório quando usado duas vezes ao dia durante quinze dias, apresentou uma diminuição mais significativa do sangramento gengival quando comparado à clorexidina $0,12 \%{ }^{52}$.

Diante do exposto, pode-se concluir que a romã apresenta bons resultados como agente anti-inflamatório, antimicrobiano e antioxidante, demonstrando eficácia no tratamento da periodontite, tornando-se um bom método alternativo para o tratamento da mesma. Todavia, mais estudos clínicos precisam ser realizados para confirmar esta hipótese.

CONCLUSÃO

Com base no exposto, malva, uva e romã possuem ação consubstanciada por testes laboratoriais e estão entre os produtos naturais com grande influência na cavidade bucal que, sob perspectivas reais de crescimento e desenvolvimento, funcionam como auxiliares no tratamento de afecções orais sendo alternativas de baixo custo e fácil acesso, uma vez que é importante que o profissional da saúde conheça a ação farmacológica dos vários medicamentos fitoterápicos, bem como seus efeitos adversos e as contraindicações. Contudo, mesmo que haja evidências sobre a eficácia destas plantas, mais estudos precisam ser realizados para confirmar estas hipóteses.

\section{REFERÊNCIAS}

1. Pasa M, Soares J, Guarim G. Estudo etnobotânico na comunidade de Conceição-Açu (alto da bacia do rio Aricá Açu, MT, Brasil). Acta bot bras. 2005;19(2):195-207.

2. Agra MF, Silva KN, Basílio IJLD, Freitas PF, Barbosa-Filho JM. Survey of medicinal plants used in the region Northeast of Brazil. Rev bras farmacogn. 2008;18(3):472-508.

3. Jesus NZT, Lima JCS, Silva RM, Espinosa MM, Martins DTO. Levantamento etnobotânico de plantas popularmente utilizadas como antiúlceras e antiinflamatórias pela comunidade de Pirizal, Nossa Senhora do Livramento-MT, Brasil. Rev bras farmacogn. 2009;19(1a):130-39.

4. Amaral JF do. Atividade antiinflamatória, antinociceptiva e gastroprotetora do óleo essencial de Croton sonderianus Muell. Arg [dissertação]. Fortaleza: Universidade Federal do Ceará. Faculdade de Medicina; 2004.

5. Rosa C, Câmara SG, Béria JU. Representações e intenção de uso da fitoterapia na atenção básica à saúde. Ciênc saúde coletiva. 2011;16(1):311-18.

6. Calixto JB. Biodiversidade como fonte de medicamentos. Cienc Cult. 2003;55(3):37-9.

7. Dewick P. Medicinal Natural Products: A Biosynthetic Approach, 3.ed. Chichester: Wiley; 2009.

8. Middleton E, Kandaswami C, Theoharides TC. The effects of plant flavonoids on mammalian cells: Implications for inflammation, heart disease, and cancer. Pharmacol Rev. 2000;52(4):673-751.

9. Simões CMO, Schenkel EP, Gosmann G, Mello JCP, Mentz LA, Petrovick PR(Orgs). Farmacognosia: da planta ao medicamento. 6.ed. Porto Alegre: Editora da UFRGS: Florianópolis: Editora da UFSC, 2010.

10. Coutinho MAS, Muzitano MF, Costa SS. Flavonoids: Potential therapeutic agents for the inflammatory process. Rev Virtual Quim. 2009;1(3):241-56.

11.Agra MF, Freitas PF, Barbosa-Filho JM. Synopsis of the plants known as medicinal and poisonous in 
Northeast of Brazil. Rev bras farmacogn. 2007;17(1):114-40.

12.Lima V, Bezerra MM, Leitão RFC, Brito GAC, Rocha FAC, Ribeiro RA. Principais mediadores inflamatórios envolvidos na fisiopatologia da periodontite- papel de moduladores farmacológicos. R Periodontia. 2008;18(3):7-19.

13. Madianos PN, Bobetsis YA, Kinane DF. Generation of inflammatory stimuli: how bacteria set up inflammatory responses in the gingiva. $\mathbf{J}$ Clin Periodontol. 2005;32(Suppl 6):57-71.

14.Sorsa T, Tjäderhane L, Salo T. Matrix metalloproteinases (MMPs) in oral diseases. Oral Dis. 2004;10(6):311-18.

15.Uğar-Çankal D, Ozmeric N. A multifaceted molecule, nitric oxide in oral and periodontal diseases. Clin Chim Acta. 2006;366(1-2):90-100.

16. Kinane DF, Preshaw PM, Loos BG, Working Group 2 of Seventh European Workshop on Periodontology. Host-response: understanding the cellular and molecular mechanisms of hostmicrobial interactions - consensus of the Seventh European Workshop on Periodontology. J Clin Periodontol. 2011;38(Suppl 11):44-8.

17. Meira ALT, Todescan SMC, Azoubel E, Bittencourt S, Azoubel MCF. Uso de antimicrobianos locais em periodontia: uma abordagem critica. R Periodontia. 2007; 17(1):83-9.

18.Filter M, Freitas EM de, Périco E. Influência de diferentes concentrações dos fitorreguladores ácido 6-benzilaminopurina e ácido naftalenoacético na propagação vegetativa de Malva sylvestris L. Rev bras plantas med. 2014;16(1):47-53.

19.Ferro D. Fitoterapia: conceitos clínicos. São Paulo: Atheneu; 2006.

20. Moreira MJS, Ferreira MBC, Hashizume LN. Avaliação In Vitro da Atividade Antimicrobiana dos Componentes de um Enxaguatório Bucal contendo Malva. Pesq Bras Odontoped Clin Integr. 2012;12(4):505-9.

21.Ecker ACL, Martins IS, Kirsch L, Lima LO, Stefenon L, Mozzini CB. Efeitos benéficos e maléficos da Malva sylvestris. J Oral Invest. 2015;4(1):39-43.

22. Ribeiro ASC, Pinto ATM, Silva DJ, Peixoto ITA. Atividade antimicrobiana de diferentes colutórios fitoterápicos. Ensaios Cienc, Cienc Biol Agrar Saúde. 2015;19(4):178-183.

23. Torres CRG, Kubo CH, Anido AA, Rodrigues JR. Agentes antimicrobianos e seu potencial de uso na Odontologia. Pos-Grad Rev Fac Odontol São José dos Campos. 2000;3(2):43-52.

24. Costa G. Efeito do extrato da casca de uva Vitis Vinífera (GSE) na pressão arterial, no perfil lipídico e glicídico e no estresse oxidativo em ratos espontaneamente hipertensos [mestrado].
Rio de Janeiro: Universidade Estadual do Rio de Janeiro, Centro Biomédico; 2008.

25.Ishimoto EY. Efeito hipolipemiante e antioxidante de subprodutos da uva em hamsters [dissertação]. São Paulo: Universidade de São Paulo, Faculdade de Saúde Pública; 2008.

26. Gris EF. Perfil fenólico e atividades antioxidante e hipolipemiante de vinhos de variedades Vitis vinifera cultivadas em São Joaquim-SC-Brasil [dissertação]. Florianópolis: Universidade Federal de Santa Catarina, Centro de Ciências Agrárias; 2010.

27. Machado MM. Desenvolvimento de uma bebida nutracêutica a partir de resíduos da produção do suco de uva: avaliação de propriedades antioxidantes e fisio-bioquímicas [tese]. Santa Maria: Universidade Federal de Santa Maria; 2010.

28. Rockenbach II, Silva GL, Rodrigues E, Gonzaga LV, Fett R. Atividade antioxidante de extratos de bagaço de uva das variedades Regente e Pinot Noir (Vitis vinifera). Rev Inst Adolfo Lutz. 2007;66(2):158-63.

29.Bozan B, Tosun G, Özcan D. Study of polyphenol content in the seeds of red grape (Vitis vinifera L.) varieties cultivated in Turkey and their antiradical activity. Food Chem. 2008;109(2):426-30.

30.Rockenbach II, Silva GL, Rodrigues E, Kuskoski EM, Fett R. Influência do solvente no conteúdo total de polifenóis, antocianinas e atividade antioxidante de extratos de bagaço de uva (Vitis vinifera) variedades Tannat e Ancelota. Ciênc Tecnol Aliment. 2008;28(Suppl):238-44.

31.Rockenbach I, Gonzaga LV, Rizelio VM, Gonçalves AES, Genovese MI, Fett R. Phenolic compounds and antioxidant activity of seed and skin extracts of red grape (Vitis vinifera and Vitis labrusca) pomace from Brazilian winemaking. Food Res Int. 2011;44(4):897-901.

32. Santos L, Morais D, Souza N, Cottica S, Boroski M, Visentainer J. Phenolic compounds and fatty acids in different parts of Vitis labrusca and V. vinifera grapes. Food Res Int. 2011; 44(5):1414-18.

33.Lachman J, Hejtmánková A, Hejtmánková K, Horníčková Š, Pivec V, Skala O et al. Towards complex utilisation of winemaking residues: characterisation of grape seeds by total phenols, tocols and essential elements content as a byproduct of winemaking. Ind Crop Prod. 2013;49:445-53.

34.Ahmadi SM, Siahsar BA. Analogy of physicochemical attributes of two grape seeds cultivars. Cien Inv Agr. 2011; 38(2):291-301.

35. Ribeiro MEM, Manfroi V. Vinho e Saúde: uma visão química. Rev Bras Vitic Etnol. 2018;2(2):91-103.

36.Ribéreau-Gayon P, Glories Y, Maujean A, 
Dubourdieu D. Handbook of enology. 2.ed. Chichester: Wiley; 2006.

37.Lorrain B, Ky I, Pechamat L, Teissedre P. Evolution of analysis of polyhenols from grapes, wines, and extracts. molecules. 2013;18(1):1076100.

38. Yoo Y, Saliba A, Prenzler P. Should Red Wine Be Considered a Functional Food?. Comprehensive Reviews in Food Science and Food Safety. 2010;9(5):530-51.

39. Çetin A, Sagdiç O. A Concise review: antioxidant effects and bioactive constituents of grape. Erciyes Med J. 2009;31(4):369-75.

40.Xia EQ, Deng GF, Guo YJ, Li HB. Biological activities of polyphenols from grapes. Int $\mathrm{J}$ Mol Sci. 2010;11(2):622-46

41. Rayyan M, Terkawi T, Abdo H, Abdel Azim D, Khalaf A, AlKhouli Z et al. Efficacy of grape seed extract gel in the treatment of chronic periodontitis: A randomized clinical study. J Investig Clin Dent. 2018;9(2):e12318.

42. Barreto VL, Feitosa AC, Araújo TJ, Chagas FK, Costa LK. Acción antimicrobiana in vitro de dentí- fricos conteniendo fitoterápicos. Av Odontoestomatol. 2005; 21(4):195-201.

43.Lorenzi H, Souza H. Plantas ornamentais no Brasil. Nova Odessa: Instituto Plantarum de Estudos da Flora; 2004.

44.Ferreira A, Ferreira M, Anjos M. Novo dicionário Aurélio da língua portuguesa. Curitiba: Positivo; 2009.

45. Vasconcelos LC, Sampaio FC, Sampaio MC, Pereira Mdo S, Higino JS, Peixoto MH. Minimum inhibitory concentration of adherence of Punica granatum Linn (pomegranate) gel against $\mathrm{S}$. mutans, S. mitis and C. albicans. Braz Dent J. 2006;17(3):223-27.

46. Menezes SM, Cordeiro LN, Viana GS. Punica granatum (pomegranate) extract is active against dental plaque. J Herb Pharmacother. 2006;6(1):79-92.

47.Barbosa M. Avaliação da atividade antimicrobiana "in vitro" da Punica granatum Linn. frente à Enterococcus faecalis isolados clinicamente [monografia de conclusão do curso]. Universidade Federal da Paraíba; 2010.

48. Catão RMR, Antunes RMP, Arruda TA, Pereira MSV, Higino JS, Alves JA et al. Atividade antimicrobiana "in vitro" do extrato etanólico de Punica granatum linn (romã) sobre isolados ambulatoriais de Staphylococcus aureus. Rev bras anal clin. 2006;38(1):111-14.

49.Jardini FA, Mancini Filho J. Avaliação da atividade antioxidante em diferentes extratos da polpa e sementes da romã (Punica granatum, L.). Rev Bras Ciênc Farm. 2007;43(1):137-47.

50.Sastravaha G, Gassmann G, Sangtherapitikul P, Grimm WD. Adjunctive periodontal treatment with Centella asiatica and Punica granatum extracts. A preliminary study. J Int Acad Periodontol. 2018;5(4):106-15.

51.Bhadbhade SJ, Acharya AB, Rodrigues SV, Thakur SL. The antiplaque efficacy of pomegranate mouthrinse. Quintessence Int. 2011;42(1):29-36.

52. Ahuja S, Dodwad V, Kukreja BJ, Mehra P, Kukreja P. A comparative evaluation of efficacy of Punica granatum and chlorhexidine on plaque and gingivitis. J Int Clin Dent Res Organ. 2011;3(1):29-32.

\section{CONFLITO DE INTERESSES}

Os autores declaram não haver conflitos de interesse.

\section{AUTOR PARA CORRESPONDENCIA}

\section{José Henrique de Araújo Cruz}

henrique_araújo1992@hotmail.com

Submetido em 25/04/2018

Aceito em 27/06/2018 